MATHEMATICS OF COMPUTATION

Volume 67, Number 224, October 1998, Pages 1577-1590

S $0025-5718(98) 00975-2$

\title{
FAST ALGORITHMS FOR DISCRETE POLYNOMIAL TRANSFORMS
}

\author{
DANIEL POTTS, GABRIELE STEIDL, AND MANFRED TASCHE
}

Dedicated to Professor G. Maess on the occasion of his 60th birthday

\begin{abstract}
Consider the Vandermonde-like matrix $\mathbf{P}:=\left(P_{k}\left(\cos \frac{j \pi}{N}\right)\right)_{j, k=0}^{N}$, where the polynomials $P_{k}$ satisfy a three-term recurrence relation. If $P_{k}$ are the Chebyshev polynomials $T_{k}$, then $\mathbf{P}$ coincides with $\mathbf{C}_{N+1}:=\left(\cos \frac{j k \pi}{N}\right)_{j, k=0}^{N}$. This paper presents a new fast algorithm for the computation of the matrixvector product $\mathbf{P a}$ in $O\left(N \log ^{2} N\right)$ arithmetical operations. The algorithm divides into a fast transform which replaces $\mathbf{P a}$ with $\mathbf{C}_{N+1} \tilde{\mathbf{a}}$ and a subsequent fast cosine transform. The first and central part of the algorithm is realized by a straightforward cascade summation based on properties of associated polynomials and by fast polynomial multiplications. Numerical tests demonstrate that our fast polynomial transform realizes $\mathbf{P a}$ with almost the same precision as the Clenshaw algorithm, but is much faster for $N \geq 128$.
\end{abstract}

\section{INTRODUCTION}

Let $w$ be a non-negative, integrable weight function with

$$
\int_{-1}^{1} w(x) \mathrm{d} x>0
$$

and let $L_{w}^{2}[-1,1]$ denote the real Hilbert space with inner product

$$
\langle f, g\rangle:=\int_{-1}^{1} w(x) f(x) g(x) \mathrm{d} x \quad\left(f, g \in L_{w}^{2}[-1,1]\right)
$$

and norm $\|\cdot\|$. As an example we consider the weight functions

$$
w(x):=\left(1-x^{2}\right)^{\lambda-1 / 2} \quad(\lambda>-1 / 2 ; x \in(-1,1)) .
$$

Let $\left\{P_{n}\right\}_{n \in \mathbb{N}_{0}}$ be a sequence of orthogonal polynomials $P_{n} \in \Pi_{n}$ with respect to $\langle\cdot, \cdot\rangle$. Here $\Pi_{n}$ denotes the set of polynomials of degree $\leq n$. Then every $P \in \Pi_{N}$ can be represented as

$$
P=\sum_{k=0}^{N} \frac{\left\langle P, P_{k}\right\rangle}{\left\|P_{k}\right\|^{2}} P_{k}
$$

Received by the editor March 15, 1996 and, in revised form, March 13, 1997.

1991 Mathematics Subject Classification. Primary 65T99, 42C10, 33C25.

Key words and phrases. Discrete polynomial transform, Vandermonde-like matrix, fast cosine transform, fast polynomial transform, Chebyshev knots, cascade summation.

(C) 1998 American Mathematical Society 
where $\left\langle P, P_{k}\right\rangle$ can be computed by a convenient quadrature rule; for example by

$$
\left\langle P, P_{k}\right\rangle=\sum_{j=0}^{2 N} w_{j}^{2 N} P\left(c_{j}^{2 N}\right) P_{k}\left(c_{j}^{2 N}\right)
$$

with the weights

$$
w_{j}^{2 N}:=\int_{-1}^{1} w(x) \frac{L(x)}{L^{\prime}\left(c_{j}^{2 N}\right)\left(x-c_{j}^{2 N}\right)} \mathrm{d} x, \quad L(x):=\prod_{j=0}^{2 N}\left(x-c_{j}^{2 N}\right),
$$

and with the Chebyshev nodes

$$
c_{j}^{N}:=\cos \frac{j \pi}{N} \quad(j=0, \ldots, N) .
$$

For $w:=1$, i.e. for Legendre polynomials $P_{k}$, the quadrature rule (1.3) coincides with the Clenshaw-Curtis quadrature with positive weights

$$
w_{j}^{2 N}:=\frac{1}{N} \varepsilon_{j}^{2 N} \sum_{l=0}^{N} \varepsilon_{l}^{N} \frac{-2}{4 l^{2}-1} c_{l j}^{N} \quad(j=0, \ldots, 2 N) .
$$

Here $\varepsilon_{0}^{N}=\varepsilon_{N}^{N}:=\frac{1}{2}$ and $\varepsilon_{j}^{N}:=1(j=1, \ldots, N-1)$. Similar, but more complicated expressions of $w_{j}^{2 N}$ can be given for the weight functions (1.1). Notice that such weights can be computed via fast cosine transforms.

Let $M, N \in \mathbb{N}$ with $M \geq N$ be given powers of 2 . We are interested in an efficient solution of the following two problems.

1. Given $a_{k} \in \mathbb{R}(k=0, \ldots, N)$ compute the discrete polynomial transform $\operatorname{DPT}(N+1, M+1): \mathbb{R}^{N+1} \rightarrow \mathbb{R}^{M+1}$ defined by

$$
\hat{a}_{j}:=\sum_{k=0}^{N} a_{k} P_{k}\left(c_{j}^{M}\right) \quad(j=0, \ldots, M) .
$$

The transform matrix $\mathbf{P}:=\left(P_{k}\left(c_{j}^{M}\right)\right)_{j, k=0}^{M, N}$ is called a Vandermonde-like matrix.

2. Given $b_{j} \in \mathbb{R}(j=0, \ldots, M)$ compute the transposed discrete polynomial transform $\operatorname{TDPT}(M+1, N+1): \mathbb{R}^{M+1} \rightarrow \mathbb{R}^{N+1}$ defined by

$$
\tilde{b}_{k}:=\sum_{j=0}^{M} b_{j} P_{k}\left(c_{j}^{M}\right) \quad(k=0, \ldots, N) .
$$

The first problem addresses the evaluation of polynomials $P \in \Pi_{N}$ given in the form (1.2) at Chebyshev nodes $c_{j}^{M}$. The second problem is concerned with the approximation of the Fourier coefficients of $P \in \Pi_{N}$ by a quadrature rule. Clearly, by (1.2) and (1.3), the problems (1.4) and (1.5) with $M=2 N, a_{k}=\left\|P_{k}\right\|^{-2}\left\langle P, P_{k}\right\rangle$ and $b_{j}=w_{j}^{2 N} P\left(c_{j}^{2 N}\right)$ are "inverse" in the sense that the corresponding transform matrices $\mathbf{P}$ and $\mathbf{P}^{\mathrm{T}}$ satisfy

$$
\begin{aligned}
& \mathbf{P} \operatorname{diag}\left(\left\|P_{k}\right\|^{-2}\right)_{k=0}^{N} \mathbf{P}^{\mathrm{T}} \operatorname{diag}\left(w_{j}^{2 N}\right)_{j=0}^{2 N}=\mathbf{I}_{2 N+1}, \\
& \mathbf{P}^{\mathrm{T}} \operatorname{diag}\left(w_{j}^{2 N}\right)_{j=0}^{2 N} \mathbf{P} \operatorname{diag}\left(\left\|P_{k}\right\|^{-2}\right)_{k=0}^{N}=\mathbf{I}_{N+1}
\end{aligned}
$$

with the $(N+1, N+1)$-identity matrix $\mathbf{I}_{N+1}$.

In general the realization of (1.4) or (1.5) requires $O(N M)$ arithmetical operations, too much for practical purposes with large $N$. Hence we look for a fast algorithm to solve our problems with only $O\left(N \log ^{2} N\right)+O(M \log M)$ arithmetical 
operations. A fast algorithm for (1.4) implies the factorization of the transform matrix $\mathbf{P}$ into a product of sparse matrices. Consequently, once a fast algorithm for (1.4) is known, a fast algorithm for the "transposed" problem (1.5) with the transform matrix $\mathbf{P}^{\mathrm{T}}$ is also available by transposing the sparse matrix product. Therefore, we restrict our attention to the fast computation of (1.4).

There are several papers addressing the problems above (see [7], [6], [9], [11], $[12],[15],[16])$. If the orthogonal polynomials are the Chebyshev polynomials of first kind

$$
T_{n}(x):=\cos (n \arccos x) \quad(x \in[-1,1]),
$$

which are orthogonal with respect to $w(x):=\left(1-x^{2}\right)^{-1 / 2}(x \in(-1,1))$, problem (1.4) can be computed via fast cosine transforms (see [17], [18], [19], [2]) in $O(M \log M)$ arithmetical operations. Hence, a straightforward idea for the fast solution of (1.4) with arbitrary orthogonal polynomials $P_{n}$ is to realize a basis exchange from $\left\{P_{n}\right\}_{n=0}^{N}$ to $\left\{T_{n}\right\}_{n=0}^{N}$ followed by a fast cosine transform.

In the case of Legendre polynomials $P_{n}$, Alpert and Rokhlin [1] have proposed an $O(N \log 1 / \varepsilon)$ basis exchange algorithm based on the approximation of the elements in the basis transform matrix. Here $\varepsilon$ denotes the desired precision.

Our direct approach computes the basis exchange with $O\left(N \log ^{2} N\right)$ arithmetical operations by a divide-and-conquer technique combined with fast polynomial multiplications. The algorithm can be designed for arbitrary polynomials $P_{n}$ satisfying a three-term recurrence relation. It requires multiplications with precomputed values of associated polynomials of $P_{n}$ occupying $O(N \log N)$ elements of storage. Numerical tests for various orthogonal polynomials, especially ultraspherical polynomials, result in small relative errors between the "exact" solution calculated in high precision arithmetic and the solution obtained by our algorithm in double precision arithmetic.

It is interesting that the "transposed" version of our (slightly modified) algorithm for the solution of the "transposed" problem (1.5) can be considered a modified Driscoll-Healy algorithm ([6], [7], [10]) in which the original fast Fourier transforms are replaced by fast cosine transforms. It is our feeling that the following approach to fast polynomial transforms is simpler and more straightforward than the original Driscoll-Healy algorithm for the problem (1.4) and (1.5).

This paper is organized as follows. Taking into account that our whole polynomial transform algorithm is based on fast realizations of different discrete cosine transforms, Section 2 deals with discrete cosine transforms. Section 3 describes our fast polynomial transform. A modified Driscoll-Healy algorithm and the relation with our algorithm is sketched in Section 4. Numerical results are presented in Section 5. Finally, Section 6 contains some concluding remarks.

\section{Discrete cosine transforms}

The heart of our fast polynomial transform consists in the fast polynomial multiplication via fast cosine transforms. Let

$$
\begin{aligned}
\mathbf{C}_{N+1} & :=\left(c_{j k}^{N}\right)_{j, k=0}^{N}, & \mathbf{D}_{N+1} & :=\operatorname{diag}\left(\varepsilon_{j}^{N}\right)_{j=0}^{N}, \\
\tilde{\mathbf{C}}_{N} & :=\left(c_{j(2 k+1)}^{2 N}\right)_{j, k=0}^{N-1}, & \tilde{\mathbf{D}}_{N} & :=\operatorname{diag}\left(\varepsilon_{j}^{N}\right)_{j=0}^{N-1} .
\end{aligned}
$$

Then the following transforms are referred to as discrete cosine transforms (DCT) of type I-III, respectively: 
$\operatorname{DCT}-\mathrm{I}(N+1): \mathbb{R}^{N+1} \rightarrow \mathbb{R}^{N+1}$ with

$$
\begin{gathered}
\hat{\mathbf{a}}:=\mathbf{C}_{N+1} \mathbf{D}_{N+1} \mathbf{a}, \\
\mathbf{a}:=\left(a_{k}\right)_{k=0}^{N}, \hat{\mathbf{a}}:=\left(\hat{a}_{j}\right)_{j=0}^{N} \in \mathbb{R}^{N+1}, \text { i.e. } \\
\hat{a}_{j}:=\sum_{k=0}^{N} \varepsilon_{k}^{N} a_{k} c_{j k}^{N}=\sum_{k=0}^{N} \varepsilon_{k}^{N} a_{k} T_{k}\left(c_{j}^{N}\right),
\end{gathered}
$$

$\operatorname{DCT}-\mathrm{II}(N): \mathbb{R}^{N} \rightarrow \mathbb{R}^{N}$ with

$$
\hat{\mathbf{b}}:=\tilde{\mathbf{C}}_{N} \mathbf{b}
$$

$\mathbf{b}:=\left(b_{k}\right)_{k=0}^{N-1}, \hat{\mathbf{b}}:=\left(\hat{b}_{j}\right)_{j=0}^{N-1} \in \mathbb{R}^{N}$, i.e.

$$
\hat{b}_{j}:=\sum_{k=0}^{N-1} b_{k} c_{j(2 k+1)}^{2 N}=\sum_{k=0}^{N-1} b_{k} T_{j}\left(c_{2 k+1}^{2 N}\right),
$$

$\operatorname{DCT}-\operatorname{III}(N): \mathbb{R}^{N} \rightarrow \mathbb{R}^{N}$ with

$$
\hat{\mathbf{b}}:=\tilde{\mathbf{C}}_{N}^{\mathrm{T}} \tilde{\mathbf{D}}_{N} \mathbf{b}
$$

i.e.

$$
\hat{b}_{j}:=\sum_{k=0}^{N-1} \varepsilon_{k}^{N} b_{k} c_{k(2 j+1)}^{2 N}=\sum_{k=0}^{N-1} \varepsilon_{k}^{N} b_{k} T_{k}\left(c_{2 j+1}^{2 N}\right) .
$$

In the following, let $N=2^{t}(t \in \mathbb{N})$. There exist various fast algorithms performing the above discrete cosine transforms with $O(N \log N)$ instead of $O\left(N^{2}\right)$ arithmetical operations. For DCT-III and DCT-II we prefer the fast algorithms in [18] because of their low arithmetical complexity and since the corresponding data permutations allow a simple, efficient implementation (see [13]). Fast algorithms for DCT-I based on [18] can be found in [2] (see also [19]). Concerning the inverse DCT's, it is easy to check (see [2]):

Lemma 2.1. It holds that

$$
\begin{aligned}
\mathbf{C}_{N+1} \mathbf{D}_{N+1} \mathbf{C}_{N+1} \mathbf{D}_{N+1} & =\frac{N}{2} \mathbf{I}_{N+1}, \\
\tilde{\mathbf{C}}_{N}^{T} \tilde{\mathbf{D}}_{N} \tilde{\mathbf{C}}_{N}=\tilde{\mathbf{C}}_{N} \tilde{\mathbf{C}}_{N}^{T} \tilde{\mathbf{D}}_{N} & =\frac{N}{2} \mathbf{I}_{N} .
\end{aligned}
$$

Hence $\left(\mathbf{C}_{N+1} \mathbf{D}_{N+1}\right)^{-1}=\frac{2}{N} \mathbf{C}_{N+1} \mathbf{D}_{N+1}$ and $\left(\tilde{\mathbf{C}}_{N}\right)^{-1}=\frac{2}{N} \tilde{\mathbf{C}}_{N}^{\mathrm{T}} \tilde{\mathbf{D}}_{N}$ such that the inverse DCT's can be computed by the same fast cosine transforms.

Let $P \in \Pi_{n}(n \in \mathbb{N})$ be given with respect to the basis of Chebyshev polynomials, i.e.

$$
P=\sum_{k=0}^{n} a_{k} T_{k}
$$

with known real coefficients $a_{k}$. Further, let $Q \in \Pi_{m}(m \in \mathbb{N})$ be a fixed polynomial with known values $Q\left(c_{2 j+1}^{2 M}\right)$ for $j=0, \ldots, M-1$, where $M=2^{s}(s \in \mathbb{N})$ with 
$M / 2 \leq m+n<M$ is chosen. Then the Chebyshev coefficients $b_{k}(k=0, \ldots, m+n)$ in

$$
R:=P Q=\sum_{k=0}^{n+m} b_{k} T_{k}
$$

can be computed in a fast way by the following procedure:

Algorithm 2.2 (Fast polynomial multiplication).

Input: $M=2^{s}(s \in \mathbb{N})$ with $M / 2 \leq m+n<M$,

$Q\left(c_{2 j+1}^{2 M}\right) \in \mathbb{R}(j=0, \ldots, M-1)$ with $Q \in \Pi_{m}$,

$a_{k} \in \mathbb{R}(k=0, \ldots, n)$.

1. Compute

$$
\left(P\left(c_{2 j+1}^{2 M}\right)\right)_{j=0}^{M-1}:=\tilde{\mathbf{C}}_{M}^{T}\left(a_{k}\right)_{k=0}^{M-1}
$$

by fast DCT-III $(M)$ of $\left(a_{k}\right)_{k=0}^{M-1}$ with $a_{k}:=0(k=n+1, \ldots, M-1)$.

2. Evaluate the $M$ products

$$
R\left(c_{2 j+1}^{2 M}\right):=P\left(c_{2 j+1}^{2 M}\right) Q\left(c_{2 j+1}^{2 M}\right) \quad(j=0, \ldots, M-1) .
$$

3. Compute

$$
\left(b_{k}\right)_{k=0}^{M-1}:=\frac{2}{M} \tilde{\mathbf{D}}_{M} \tilde{\mathbf{C}}_{M}\left(R\left(c_{2 j+1}^{2 M}\right)\right)_{j=0}^{M-1}
$$

by fast DCT-II $(M)$ of $\left(R\left(c_{2 j+1}^{2 M}\right)\right)_{j=0}^{M-1}$.

Output: $b_{k}(k=0, \ldots, m+n)$.

The fast DCT-III $\left(2^{s}\right)$ computed by [18] requires $2^{s-1} s$ multiplications and $2^{s-1}(3 s-2)+1$ additions. Hence, Algorithm 2.2 realizes the polynomial multiplication of $P \in \Pi_{n}$ and $Q \in \Pi_{m}$ with respect to the basis of Chebyshev polynomials in $2^{s}(s+2)+2$ multiplications and $2^{s}(3 s-2)+2$ additions.

Remark 2.3. A similar algorithm for the fast polynomial multiplication can be derived involving DCT-I instead of DCT-II and DCT-III, if the values $Q\left(c_{j}^{M}\right)$ $(j=0, \ldots, M)$ are known (see [2]).

\section{FAST POLYNOMIAL TRANSFORM}

Let $\left\{P_{n}\right\}_{n \in \mathbb{N}_{0}}$ be a sequence of polynomials defined by the three-term recurrence relation

$$
\begin{aligned}
P_{-1}(x) & :=0, \quad P_{0}(x):=1 \\
P_{n}(x) & =\left(\alpha_{n} x+\beta_{n}\right) P_{n-1}(x)+\gamma_{n} P_{n-2}(x) \quad(n=1,2, \ldots)
\end{aligned}
$$

with $\alpha_{n}, \beta_{n}, \gamma_{n} \in \mathbb{R}$ and $\alpha_{n}>0, \gamma_{n} \neq 0(n \in \mathbb{N})$. By Favard's theorem, $\left\{P_{n}\right\}_{n=0}^{\infty}$ is an orthogonal polynomial sequence with respect to some quasi-definite moment functional (see [4], Theorem 4.4). In particular, we consider the Chebyshev polynomials $T_{n}$ with

$$
\begin{aligned}
& T_{0}(x):=1, \quad T_{1}(x):=x, \\
& T_{n}(x)=2 x T_{n-1}(x)-T_{n-2}(x) \quad(n=2,3, \ldots) .
\end{aligned}
$$

Shifting the index $n$ in (3.1) by $c \in \mathbb{N}_{0}$, we obtain the associated polynomials $P_{n}(\cdot, c)$ of $P_{n}$ defined by

$$
\begin{aligned}
P_{-1}(x, c) & :=0, \quad P_{0}(x, c):=1, \\
P_{n}(x, c) & :=\left(\alpha_{n+c} x+\beta_{n+c}\right) P_{n-1}(x, c)+\gamma_{n+c} P_{n-2}(x, c) \quad(n=1,2, \ldots) .
\end{aligned}
$$


Now induction yields (see [3])

Lemma 3.1. For $c, n \in \mathbb{N}_{0}$, it holds

$$
P_{c+n}(x)=P_{n}(x, c) P_{c}(x)+\gamma_{c+1} P_{n-1}(x, c+1) P_{c-1}(x) .
$$

Lemma 3.1 implies

$$
\left(\begin{array}{c}
P_{c+n} \\
P_{c+n+1}
\end{array}\right)=\mathbf{U}_{n}(\cdot, c)^{\mathrm{T}}\left(\begin{array}{c}
P_{c-1} \\
P_{c}
\end{array}\right)
$$

with

$$
\mathbf{U}_{n}(x, c):=\left(\begin{array}{cc}
\gamma_{c+1} P_{n-1}(x, c+1) & \gamma_{c+1} P_{n}(x, c+1) \\
P_{n}(x, c) & P_{n+1}(x, c)
\end{array}\right) .
$$

Let $N=2^{t}$ and $M=2^{s}(s, t \in \mathbb{N} ; s \geq t)$ be given. Consider

$$
P:=\sum_{k=0}^{N} a_{k} P_{k} \in \Pi_{N}
$$

with known real coefficients $a_{k}$. Our concern is the fast evaluation of $P\left(c_{j}^{M}\right)$ $(j=0, \ldots, M)$ with $O\left(N \log ^{2} N\right)+O(M \log M)$ instead of $O(M N)$ arithmetical operations. The main part of our algorithm realizes the basis exchange from $\left\{P_{k}\right\}_{k=0}^{N}$ to $\left\{T_{k}\right\}_{k=0}^{N}$ in $\Pi_{N}$ and produces the Chebyshev coefficients $\tilde{a}_{k}$ in

$$
P=\sum_{k=0}^{N} \tilde{a}_{k} T_{k}
$$

Knowing these Chebyshev coefficients $\tilde{a}_{k}$, the values $P\left(c_{j}^{M}\right)(j=0, \ldots, M)$ can be computed via fast DCT-I $(M+1)$ in $O(M \log M)$ arithmetical operations in a final step (see [19], [2])

$$
\left(P\left(c_{j}^{M}\right)\right)_{j=0}^{M}=\mathbf{C}_{M}\left(\tilde{a}_{k}\right)_{k=0}^{M},
$$

where we have to set $\tilde{a}_{k}:=0$ for $k=N+1, \ldots, M$.

Let us turn to the basis exchange. In the initial step we use (3.1) and the fact that $T_{1}(x)=x$ to obtain

$$
P=\sum_{k=0}^{N-1} a_{k}^{(0)} P_{k}=\sum_{k=0}^{N / 4-1}\left(\sum_{l=0}^{3} a_{4 k+l}^{(0)} P_{4 k+l}\right)
$$

with

$$
\begin{aligned}
a_{k}^{(0)}(x) & :=a_{k} \quad(k=0, \ldots, N-3), \\
a_{N-2}^{(0)}(x) & :=a_{N-2}+\gamma_{N-1} a_{N}, \\
a_{N-1}^{(0)}(x) & :=a_{N-1}+\beta_{N-1} a_{N}+\alpha_{N-1} a_{N} T_{1}(x) .
\end{aligned}
$$

Now we proceed by cascade summation as shown in Figure 1. By (3.2) with $n=1$ and $c=4 k+1(k=0, \ldots, N / 4-1)$ it follows that

$$
\left(a_{4 k+2}^{(0)}, a_{4 k+3}^{(0)}\right)\left(\begin{array}{c}
P_{4 k+2} \\
P_{4 k+3}
\end{array}\right)=\left(a_{4 k+2}^{(0)}, a_{4 k+3}^{(0)}\right) \mathbf{U}_{1}(\cdot, 4 k+1)^{\mathrm{T}}\left(\begin{array}{c}
P_{4 k} \\
P_{4 k+1}
\end{array}\right) .
$$


Thus

$$
P=\sum_{k=0}^{N / 4-1}\left(a_{4 k}^{(1)} P_{4 k}+a_{4 k+1}^{(1)} P_{4 k+1}\right)
$$

with

$$
\left(\begin{array}{c}
a_{4 k}^{(1)} \\
a_{4 k+1}^{(1)}
\end{array}\right):=\left(\begin{array}{c}
a_{4 k}^{(0)} \\
a_{4 k+1}^{(0)}
\end{array}\right)+\mathbf{U}_{1}(\cdot, 4 k+1)\left(\begin{array}{c}
a_{4 k+2}^{(0)} \\
a_{4 k+3}^{(0)}
\end{array}\right) .
$$

The degree of the polynomial products in (3.6) is at most 3 such that their computation with respect to the Chebyshev polynomials can be realized via Algorithm 2.2 with $M=4$. Consequently, the evaluation of the Chebyshev coefficients of the polynomials $a_{4 k}^{(1)}, a_{4 k+1}^{(1)} \in \Pi_{3}(k=0, \ldots, N / 4-1)$ in step 1 requires $11 N$ multiplications and $12 N$ additions.

We continue in the obvious manner. In step $\tau(1<\tau<t)$ we compute by $(3.2)$ with $n=2^{\tau}-1$ the Chebyshev coefficients of the polynomials $a_{2^{\tau+1} k}^{(\tau)}, a_{2^{\tau+1} k+1}^{(\tau)} \in$ $\Pi_{2^{\tau+1}-1}\left(k=0, \ldots, N / 2^{\tau+1}-1\right)$ defined by

$$
\left(\begin{array}{c}
a_{2^{\tau+1} k}^{(\tau)} \\
a_{2^{\tau+1} k+1}^{(\tau)}
\end{array}\right):=\left(\begin{array}{c}
a_{2^{\tau+1} k}^{(\tau-1)} \\
a_{2^{\tau+1} k+1}^{(\tau-1)}
\end{array}\right)+\mathbf{U}_{2^{\tau}-1}\left(\cdot, 2^{\tau+1} k+1\right)\left(\begin{array}{c}
a_{2^{\tau+1} k+2^{\tau}}^{(\tau-1)} \\
a_{2^{\tau+1} k+2^{\tau}+1}^{(\tau-1)}
\end{array}\right),
$$

where we apply Algorithm 2.2 (with $M=2^{\tau+1}$ ) for the polynomial products. Assume that the $4 N$ values $U_{2^{\tau}-1}\left(c_{2 l+1}^{2^{\tau+2}}, 2^{\tau+1} k+1\right)$ for $k=0, \ldots, N / 2^{\tau+1}$ and $l=$ $0, \ldots, 2^{\tau+1}-1$ were precomputed by the Clenshaw algorithm (see [5] or [20], pp. 165 - 172). Then step $\tau$ requires $\left(2 \tau+8+2^{1-\tau}\right) N$ multiplications and $\left(6 \tau+5+2^{1-\tau}\right) N$ additions, and results in

$$
P=\sum_{k=0}^{N / 2^{\tau+1}-1}\left(a_{2^{\tau+1} k}^{(\tau)} P_{2^{\tau+1} k}+a_{2^{\tau+1} k+1}^{(\tau)} P_{2^{\tau+1} k+1}\right) .
$$

After the step $t-1$, our cascade summation arrives at

$$
P=a_{0}^{(t-1)} P_{0}+a_{1}^{(t-1)} P_{1} .
$$

Now $P_{0}(x)=1, P_{1}(x)=\alpha_{1} x+\beta_{1}$ and

$$
x T_{0}(x)=T_{1}(x), \quad x T_{n}(x)=\frac{1}{2}\left(T_{n+1}(x)+T_{n-1}(x)\right) \quad(n=1,2, \ldots) .
$$

Hence, if

$$
a_{1}^{(t-1)}=\sum_{n=0}^{N-1} a_{1, n}^{(t-1)} T_{n}
$$

then

$$
a_{1}^{(t)}:=a_{1}^{(t-1)} P_{1}=\sum_{n=0}^{N} a_{1, n}^{(t)} T_{n}
$$

with

$$
\left(a_{1, n}^{(t)}\right)_{n=0}^{N}=\left(\alpha_{1} \mathbf{T}_{N+1}^{\mathrm{T}}+\beta_{1} \mathbf{I}_{N+1}\right)\left(a_{1, n}^{(t-1)}\right)_{n=0}^{N}
$$




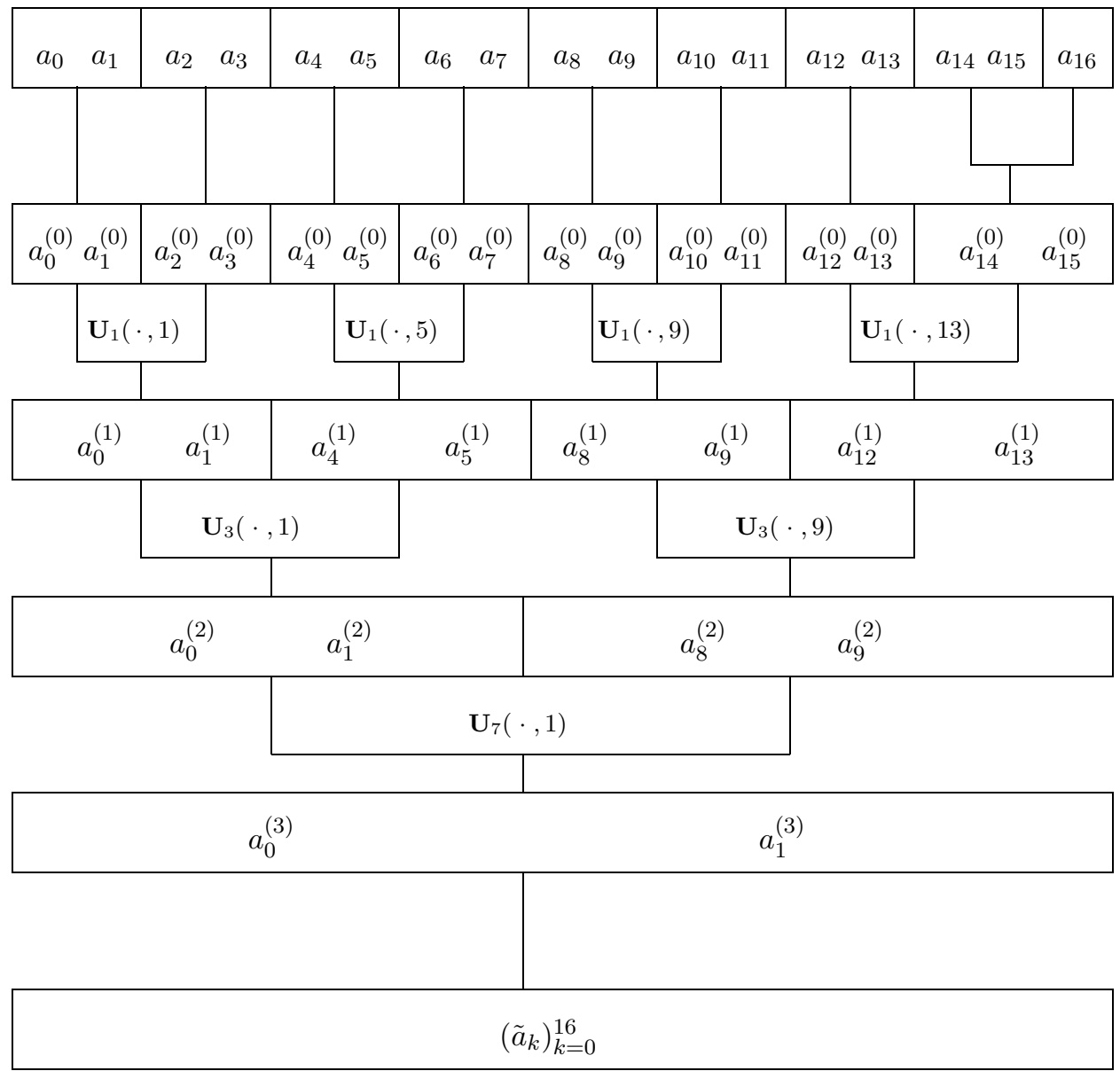

Figure 1. Cascade summation for the computation of the basis exchange in the case $N=16$

where we set $a_{1, N}^{(t-1)}:=0$ and where $\mathbf{T}_{N+1}$ is the tridiagonal $(N+1, N+1)$-matrix

$$
\mathbf{T}_{N+1}:=\left(\begin{array}{ccccc}
0 & 1 & & & \\
1 / 2 & 0 & 1 / 2 & & \\
& \ddots & \ddots & \ddots & \\
& & 1 / 2 & 0 & 1 / 2 \\
& & & 1 & 0
\end{array}\right) .
$$

This leads to

$$
P=a_{0}^{(t-1)}+a_{1}^{(t)}
$$

and the final addition of the Chebyshev coefficients of $a_{0}^{(t-1)}$ and $a_{1}^{(t)}$ yields the desired Chebyshev coefficients of $P$, i.e.

$$
\left(\tilde{a}_{n}\right)_{n=0}^{N}=\left(a_{0, n}^{(t-1)}\right)_{n=0}^{N}+\left(a_{1, n}^{(t)}\right)_{n=0}^{N} .
$$

We summarize: 
Algorithm 3.2 (Fast polynomial transform).

Input: $N=2^{t}, M=2^{s}(s, t \in \mathbb{N} ; s \geq t)$,

$$
\begin{aligned}
& a_{k} \in \mathbb{R}(k=0, \ldots, N), \\
& \mathbf{U}_{2^{\tau}-1}\left(c_{2 l+1}^{2^{\tau+2}}, 2^{\tau+1} k+1\right)\left(\tau=1, \ldots, t-1 ; k=0, \ldots, 2^{t-\tau-1}\right. \\
& \left.l=0, \ldots, 2^{\tau+1}-1\right) .
\end{aligned}
$$

Step 0. Compute $a_{k}^{(0)}(x)\left(k=0, \ldots, 2^{t}-1\right)$ by $(3.5)$.

For $\tau=1, \ldots, t-1$ do

Step $\tau$. For every $k=0, \ldots, 2^{t-\tau-1}-1$ form (3.7) by Algorithm 2.2

for the fast polynomial multiplications.

Step t. Compute $\tilde{a}_{n}(n=0, \ldots, N)$ by (3.8) and (3.10).

Compute (3.4) by fast DCT-I $(M+1)$.

Output: $P\left(c_{j}^{M}\right)(j=0, \ldots, M)$.

In summary, we have to store the $4 N(\log N-1)$ precomputed elements of the matrices $\mathbf{U}$. Counting the arithmetical operations in each step, we verify that the whole basis exchange algorithm requires $N \log ^{2} N+7 N \log N+O(N)$ multiplications and $3 N \log ^{2} N+2 N \log N+O(N)$ additions. Finally, the computation of (3.4) by the fast DCT-I $(M+1)$ takes $\frac{1}{2} M \log M+O(M)$ multiplications and $\frac{3}{2} M \log M+O(M)$ additions. The whole fast polynomial transform becomes more efficient than the Clenshaw algorithm for $N \geq 128$.

A fast algorithm for (1.5), i.e., for the multiplication with $\mathbf{P}^{\mathrm{T}}$, can be obtained immediately by "reversing" Algorithm 3.2. In other words, we simply have to reverse the direction of the arrows in the flowgraph of Algorithm 3.2.

There already exists an $O\left(N \log ^{2} N\right)$ algorithm for the problem (1.4) as well as (1.5), which was originally formulated by Driscoll and Healy [6] with respect to Legendre polynomials and was generalized to arbitrary polynomials satisfying a three-term recurrence relation in [7], [10]. The following section briefly describes the relation between our algorithm and the Driscoll-Healy algorithm.

\section{Modified Driscoll-Healy Algorithm}

In the following, we modify the Driscoll-Healy algorithm by replacing the original fast Fourier transforms by fast cosine transforms which seem to be more natural in the context of the algorithm. As a consequence, the modified algorithm is simpler, requires fewer arithmetical operations, and avoids the arithmetic with complex numbers.

Remark 4.1. The original Driscoll-Healy algorithm uses properties of circulant matrices. Our modified algorithm utilizes the fact that "circulant matrices related to the DCT-I" possess similar properties (see [2]). The matrix $\mathbf{T}_{N+1}$ defined by (3.9) is called the basic circulant matrix related to DCT-I $(N+1)$ since

$$
\frac{N}{2} \mathbf{C}_{N+1}^{\mathrm{I}} \mathbf{T}_{N+1} \mathbf{C}_{N+1}^{\mathrm{I}}=\boldsymbol{\Gamma}_{N+1}
$$

with $\mathbf{C}_{N+1}^{\mathrm{I}}:=\mathbf{C}_{N+1} \mathbf{D}_{N+1}$ and $\boldsymbol{\Gamma}_{N+1}:=\operatorname{diag}\left(c_{j}^{N}\right)_{j=0}^{N}$. Let $\mathbf{T}_{N+1,0}:=\mathbf{I}_{N+1}$, $\mathbf{T}_{N+1,1}:=\mathbf{T}_{N+1}$ and

$$
\mathbf{T}_{N+1, n}=2 \mathbf{T}_{N+1} \mathbf{T}_{N+1, n-1}-\mathbf{T}_{N+1, n-2} \quad(n=2,3, \ldots, N) .
$$


Then $\mathbf{T}_{N+1, N}=\left(\delta_{j, N-k}\right)_{j, k=0}^{N}$ and

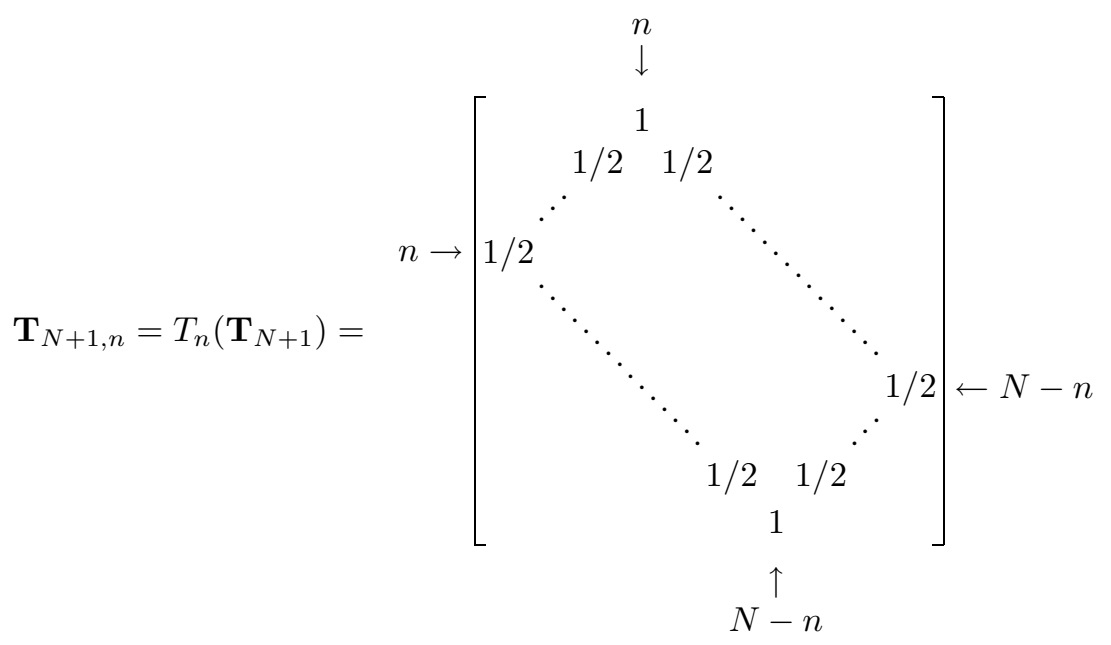

for $n=1, \ldots, N-1$. The matrices diagonalizable by $\mathbf{C}_{N+1}^{\mathrm{I}}$ can be written as

$$
\mathbf{P}=\sum_{j=0}^{N} \varepsilon_{j}^{N} a_{j} \mathbf{T}_{N+1, j} \quad\left(a_{j} \in \mathbb{R}\right)
$$

and form a commutative algebra $\mathcal{P}$ over $\mathbb{R}$ with respect to the ordinary matrix operations. It holds

$$
\frac{N}{2} \mathbf{C}_{N+1}^{\mathrm{I}} \mathbf{P} \mathbf{C}_{N+1}^{\mathrm{I}}=P\left(\boldsymbol{\Gamma}_{N+1}\right),
$$

where

$$
P:=\sum_{j=0}^{N} \varepsilon_{j}^{N} a_{j} T_{j}
$$

denotes the polynomial associated with $\mathbf{P}$. Based on the fact that the Chebyshev knots $c_{k}^{N}(k=0, \ldots, N)$ are the zeros of $\left(1-x^{2}\right) U_{N-1}(x)$, the relation between circulant matrices related to DCT-I and polynomials is determined by the isomorphism of the algebras

$$
\mathcal{P} \simeq \mathbb{R}[x] /\left(\left(1-x^{2}\right) U_{N-1}(x)\right) .
$$

Here $U_{N-1}$ is the $(N-1)$-th Chebyshev polynomial of second kind.

Let the polynomials $P_{l}$ satisfy the three-term recurrence relation (3.1). Consider (1.5) with $M=N=2^{t}(t \in \mathbb{N})$, i.e.

$$
\tilde{b}_{l}:=\sum_{j=0}^{N} \varepsilon_{j}^{N} d_{j} P_{l}\left(c_{j}^{N}\right) \quad(l=0, \ldots, N)
$$

with given $d_{j}:=\left(\varepsilon_{j}^{N}\right)^{-1} b_{j} \in \mathbb{R}$. Following the lines of [6], we define

$$
z(k, l):=\sum_{j=0}^{N} \varepsilon_{j}^{N} d_{j} c_{j k}^{N} P_{l}\left(c_{j}^{N}\right) \quad(k, l=0, \ldots, N)
$$


and $\mathbf{z}_{l}:=(z(k, l))_{k=0}^{N}$. We are interested in the efficient computation of $z(0, l)=\tilde{b}_{l}$ $(l=0, \ldots, N)$. By Lemma 2.1 we observe that

$$
\begin{aligned}
\mathbf{z}_{0} & =\mathbf{C}_{N+1}^{\mathrm{I}}\left(d_{j}\right)_{j=0}^{N}, \\
\mathbf{z}_{l} & =\mathbf{C}_{N+1}^{\mathrm{I}}\left(\left(P_{l}\left(c_{j}^{N}\right)\right)_{j=0}^{N} \circ\left(d_{j}\right)_{j=0}^{N}\right) \\
& =\frac{2}{N} \mathbf{C}_{N+1}^{\mathrm{I}} P_{l}\left(\boldsymbol{\Gamma}_{N+1}\right) \mathbf{C}_{N+1}^{\mathrm{I}} \mathbf{z}_{0},
\end{aligned}
$$

where $\circ$ denotes the componentwise multiplication. Especially, we obtain with $P_{1}(x)=\alpha_{1} x+\beta_{1}$ by (4.1) and (4.3) that

$$
\begin{aligned}
\mathbf{z}_{1} & =\frac{2}{N} \mathbf{C}_{N+1}^{\mathrm{I}}\left(\alpha_{1} \boldsymbol{\Gamma}_{N+1}+\beta_{1} \mathbf{I}_{N+1}\right) \mathbf{C}_{N+1}^{\mathrm{I}} \mathbf{z}_{0} \\
& =\left(\alpha_{1} \mathbf{T}_{N+1}+\beta_{1} \mathbf{I}_{N+1}\right) \mathbf{z}_{0}
\end{aligned}
$$

(compare with (3.8)). Using (4.2) and (3.2), we compute $\mathbf{z}_{N / 2}$ and $\mathbf{z}_{N / 2+1}$ by

$$
\left(\begin{array}{c}
\mathbf{z}_{N / 2} \\
\mathbf{z}_{N / 2+1}
\end{array}\right)=\frac{2}{N}\left(\mathbf{C}_{N+1}^{\mathrm{I}} \oplus \mathbf{C}_{N+1}^{\mathrm{I}}\right) \mathbf{U}_{N / 2-1}\left(\boldsymbol{\Gamma}_{N+1}, 1\right)^{\mathrm{T}}\left(\mathbf{C}_{N+1}^{\mathrm{I}} \oplus \mathbf{C}_{N+1}^{\mathrm{I}}\right)\left(\begin{array}{l}
\mathbf{z}_{0} \\
\mathbf{z}_{1}
\end{array}\right)
$$

and form the truncated vectors

$$
\mathbf{z}_{l}^{(1)}:=(z(k, l))_{k=0}^{N / 2} \quad(l=0,1, N / 2, N / 2+1) .
$$

This is the result of step 1 of our modified Driscoll-Healy algorithm. Compare with step $t-1$ of Algorithm 3.2. Note that $\mathbf{U}_{N / 2-1}\left(\boldsymbol{\Gamma}_{N+1}, 1\right)$ is a block-diagonal $(2 N+2,2 N+2)$-matrix.

Now we continue in a similar manner as in [6], [7] but with respect to circulant matrices related to the DCT-I. Take into consideration that the description of the following steps involves some more ideas than step 1. The resulting modified Driscoll-Healy algorithm does not agree with the "transposed" version of Algorithm 3.2, but it can be considered as a "transposed" version of a modified Algorithm 3.2 in which the fast polynomial multiplications are realized by DCT-I instead of DCT-II and DCT-III, as mentioned in Remark 2.3. However, in our opinion the derivation of the (modified) Driscoll-Healy algorithm is less straightforward than the development of Algorithm 3.2.

\section{Numerical tests}

Algorithm 3.2 was implemented in C and tested on a Sun SPARCstation 20 for various ultraspherical polynomials. The corresponding fast cosine transforms were described in detail in [18], [2].

Example 5.1. We consider the ultraspherical polynomials $P_{n}^{\lambda}(\lambda>-1 / 2)$ given by

$$
\begin{aligned}
P_{-1}^{\lambda}(x) & :=0, \quad P_{0}^{\lambda}(x):=1, \\
P_{n}^{\lambda}(x) & :=\frac{2(n+\lambda-1)}{n} x P_{n-1}^{\lambda}(x)-\frac{n+2 \lambda-2}{n} P_{n-2}^{\lambda}(x) \quad(n=1,2, \ldots) .
\end{aligned}
$$

These polynomials are orthogonal with respect to the weight function (1.1). For $\lambda=\frac{n-2}{2}$, the ultraspherical polynomials are the zonal spherical polynomials of $S^{n-1}$ with respect to $\mathrm{SO}(n) / \mathrm{SO}(n-1)$. For the 2 -sphere $S^{2}$, i.e. for $\lambda=1 / 2$, 


\section{TABLE 1}

\begin{tabular}{|c|c|c|c|c|}
\hline$N$ & $\lambda$ & $a_{k}$ & $\varepsilon(\mathrm{CA})$ & $\varepsilon(\mathrm{FPT})$ \\
\hline \hline 256 & 0.5 & $1 /(k+1)$ & $3.88 E-16$ & $3.77 E-13$ \\
512 & 0.5 & $1 /(k+1)$ & $1.59 E-14$ & $5.73 E-12$ \\
1024 & 0.5 & $1 /(k+1)$ & $4.21 E-13$ & $8.98 E-12$ \\
2048 & 0.5 & $1 /(k+1)$ & $2.11 E-12$ & $3.19 E-11$ \\
\hline 256 & 1.5 & $1 /(k+1)$ & $1.88 E-13$ & $8.36 E-13$ \\
512 & 1.5 & $1 /(k+1)$ & $6.12 E-13$ & $1.29 E-11$ \\
1024 & 1.5 & $1 /(k+1)$ & $1.26 E-12$ & $8.00 E-11$ \\
\hline 256 & 5 & $1 /(k+1)$ & $1.15 E-13$ & $2.72 E-13$ \\
512 & 5 & $1 /(k+1)$ & $5.15 E-13$ & $4.37 E-12$ \\
1024 & 5 & $1 /(k+1)$ & $1.04 E-12$ & $5.18 E-12$ \\
\hline 256 & 2 & 1 & $2.44 E-13$ & $7.52 E-13$ \\
512 & 2 & 1 & $8.61 E-13$ & $6.61 E-12$ \\
1024 & 2 & 1 & $1.71 E-12$ & $4.82 E-12$ \\
\hline
\end{tabular}

the ultraspherical polynomials are the Legendre polynomials. For given $a_{k} \in \mathbb{R}$ $(k=0, \ldots, N)$ we compute

$$
\hat{a}_{j}=\sum_{k=0}^{N} a_{k} P_{k}^{\lambda}\left(c_{j}^{N}\right) \quad(j=0, \ldots, N)
$$

by the Clenshaw algorithm $(\mathrm{CA})$ in double precision arithmetic, the Clenshaw algorithm realized in Maple with high precision arithmetic of 64 digits $\left(\mathrm{CA}_{64}\right)$, and by our fast polynomial transform (FPT) in double precision arithmetic. Table 1 compares the results for different transform lengths $N$ ranging between 256 and 2048 and various parameters $\lambda$. The third column of the table contains the given coefficients $a_{k}$, while the fourth and last columns contain the relative error $\varepsilon(\mathrm{CA})$ of the Clenshaw algorithm defined by

$$
\varepsilon(\mathrm{CA}):=\max _{0 \leq j \leq N}\left|\hat{a}_{j}(\mathrm{CA})-\hat{a}_{j}\left(\mathrm{CA}_{64}\right)\right| / \max _{0 \leq j \leq N}\left|\hat{a}_{j}\left(\mathrm{CA}_{64}\right)\right|
$$

and the relative error of Algorithm 3.2 given by

$$
\varepsilon(\mathrm{FPT}):=\max _{0 \leq j \leq N}\left|\hat{a}_{j}(\mathrm{FPT})-\hat{a}_{j}\left(\mathrm{CA}_{64}\right)\right| / \max _{0 \leq j \leq N}\left|\hat{a}_{j}\left(\mathrm{CA}_{64}\right)\right| .
$$

Here $\hat{a}_{j}(\mathrm{CA}), \hat{a}_{j}\left(\mathrm{CA}_{64}\right)$ and $\hat{a}_{j}(\mathrm{FPT})$ denote the corresponding results of (5.1) using $\mathrm{CA}, \mathrm{CA}_{64}$ and $\mathrm{FPT}$, respectively.

Note that both the Clenshaw algorithm and fast polynomial transform realize the problem (5.1) with almost the same precision, but our method is much faster than the Clenshaw algorithm as shown in Example 5.2.

Example 5.2. As in Example 5.1 we use ultraspherical polynomials. It is wellknown that the Clenshaw algorithm requires $N^{2}$ multiplications and $3 N^{2}$ additions. Algorithm 3.2 is significantly faster for large $N \geq 128$. The third and fourth columns of Table 2 list the CPU-times $t(\mathrm{CA})$ and $t(\mathrm{FPT})$ (in seconds) for the Clenshaw algorithm and for Algorithm 3.2. The last column contains the relative error

$$
\tilde{\varepsilon}(\mathrm{FPT}):=\max _{0 \leq j \leq N}\left|\hat{a}_{j}(\mathrm{FPT})-\hat{a}_{j}(\mathrm{CA})\right| / \max _{0 \leq j \leq N}\left|\hat{a}_{j}(\mathrm{CA})\right|
$$


TABLE 2

\begin{tabular}{|c|c|c|c|c|}
\hline$N$ & $\lambda$ & $t(\mathrm{CA})$ & $t(\mathrm{FPT})$ & $\tilde{\varepsilon}(\mathrm{FPT})$ \\
\hline \hline 128 & 0.5 & 0.05 & 0.04 & $3.59 E-14$ \\
256 & 0.5 & 0.21 & 0.07 & $4.35 E-12$ \\
512 & 0.5 & 0.82 & 0.19 & $4.93 E-12$ \\
1024 & 0.5 & 3.27 & 0.39 & $5.78 E-11$ \\
2048 & 0.5 & 13.70 & 0.85 & $2.09 E-10$ \\
4096 & 0.5 & 55.41 & 1.92 & $1.04 E-09$ \\
8192 & 0.5 & 220.05 & 4.26 & $5.04 E-08$ \\
\hline 4096 & 2.5 & 55.43 & 1.91 & $1.72 E-09$ \\
\hline 4096 & 4.0 & 55.42 & 1.91 & $6.41 E-10$ \\
\hline 4096 & 5.0 & 55.42 & 1.92 & $3.35 E-10$ \\
\hline
\end{tabular}

Here the original coefficients $a_{k}(k=0, \ldots, N)$ are randomly distributed in the interval $[-0.5,0.5]$.

\section{Conclusions}

A motivation to consider the discrete polynomial transforms (1.4) and (1.5) with respect to the $2 N+1$ Chebyshev nodes $c_{j}^{2 N}(j=0, \ldots, 2 N)$ arises from the Clenshaw-Curtis quadrature which seems to be very useful for the computation of the Fourier coefficients in (1.2) in the case of Legendre polynomials $P_{n}$, i.e. $w=1$. However, other quadrature rules may be of interest. Gaussian quadrature reduces the number of required nodes in (1.3) from $2 N+1$ to $N+1$. Hence a natural question is how to compute

$$
\sum_{k=0}^{N} a_{k} P_{k}\left(x_{j}^{M}\right) \quad(j=0, \ldots, M ; M \geq N)
$$

for arbitrary $x_{j}^{M} \in[-1,1]$ and $a_{k} \in \mathbb{R}$ in an efficient way. The heart of our method, the basis exchange in Algorithm 3.2 is independent of the choice of knots $x_{j}^{M}$. So it remains to perform the final step of our algorithm, the computation of

$$
\sum_{k=0}^{N} \tilde{a}_{k} T_{k}\left(x_{j}^{M}\right) \quad(j=0, \ldots, M),
$$

in a fast way. One possibility for realizing (6.1) in $O\left(M \log ^{2} M\right)$ arithmetical operations based on a (heuristic) stabilization of the Borodin-Munro algorithm was suggested in [14]. We prefer the application of the fast adaptive multipole method (see [8]), which computes (6.1) in $O(M \log 1 / \varepsilon)$ arithmetical operations, where $\varepsilon$ denotes the desired precision. This approach seems to be interesting in connection with fast Fourier transforms on spheres and on distance transitive graphs, too (see [7]). The results will be presented in a forthcoming paper.

\section{ACKNOWLEDGEMENT}

The authors thank the referee for helpful comments and for pointing out the references [10] and [12]. 


\section{REFERENCES}

[1] B. K. Alpert and V. Rokhlin, A fast algorithm for the evaluation of Legendre expansions, SIAM J. Sci. Statist. Comput. 12 (1991), 158 - 179. MR 91i:65042

[2] G. Baszenski and M. Tasche, Fast polynomial multiplication and convolutions related to the discrete cosine transform, Linear Algebra Appl. 252 (1997), 1 - 25. CMP 97:06

[3] S. Belmehdi, On the associated orthogonal polynomials, J. Comput. Appl. Math. 32 (1991), 311 - 319. MR 92e:33007

[4] T. S. Chihara, An Introduction to Orthogonal Polynomials, Gordon and Breach, New York, 1978. MR 58:1979

[5] C. W. Clenshaw, A note on the summation of Chebyshev series, Math. Comp. 9 (1955), 118 - 120. MR 17:194e

[6] J. R. Driscoll and D.M. Healy, Computing Fourier transforms and convolutions on the 2sphere, Adv. in Appl. Math. 15 (1994), 202 - 240. MR 95h:65108

[7] J. R. Driscoll, D. M. Healy and D. Rockmore, Fast discrete polynomial transforms with applications to data analysis for distance transitive graphs, SIAM J. Sci. Comput. 26 (1997), 1066-1099. CMP 97:15

[8] A. Dutt, M. Gu and V. Rokhlin, Fast algorithms for polynomial interpolation, integration and differentiation, SIAM J. Numer. Anal. 33 (1996), 1689-1711. MR 97h:65015

[9] W. Gautschi, The condition of Vandermonde-like matrices involving orthogonal polynomials, Linear Algebra Appl. 52/53 (1983), 293 - 300. MR 84i:65043

[10] D. M. Healy, S. Moore and D. Rockmore, Efficiency and stability issues in the numerical convolution of Fourier transforms and convolutions on the 2-sphere, Technical Report, Dartmouth College, 1994.

[11] N. J. Higham, Fast solution of Vandermonde-like systems involving orthogonal polynomials, IMA J. Numer. Anal. 8 (1988), 473 - 486. MR 89k:65067

[12] D. Maslen, A polynomial approach to orthogonal polynomial transforms, Research Report, Max-Planck-Institute of Mathematics, Bonn, 1994.

[13] S. S. B. Moore, Efficient stabilization methods for fast polynomial transforms, Thesis, Dartmouth College, 1994.

[14] S. S. B. Moore, D.M. Healy and D.N. Rockmore, Symmetry stabilization for fast discrete monomial transforms and polynomial evaluation, Linear Algebra Appl. 192 (1993), 249 299. MR 94g:65148

[15] S. A. Orszag, Fast eigenfunction transforms, in: Science and Computers (G.C. Rota, ed.), Academic Press, New York, 1986, 23 - 30.

[16] V. Pan, Fast evaluation and interpolation at the Chebyshev sets of points, Appl. Math. Lett. 34 (1989), 255 - 258. CMP 21:17

[17] K. R. Rao and P. Yip, Discrete Cosine Transform, Academic Press, Boston, 1990. MR 92b:94003

[18] G. Steidl, Fast radix $-p$ discrete cosine transform, Appl. Algebra in Engrg. Comm. Comput. 3 (1992), 39 - 46. MR 95m:65221

[19] G. Steidl and M. Tasche, A polynomial approach to fast algorithms for discrete Fourier-cosine and Fourier-sine transforms, Math. Comp. 56 (1991), 281 - 296. MR 91h:65225

[20] J. Wimp, Computation with Recurrence Relations, Pitman Press, Boston, 1984. MR 85f:65001

Fachbereich Mathematik, Universität Rostock, D-18051 Rostock

E-mail address: daniel.potts@stud.uni-rostock.de

FAKUltät FÜr Mathematik Und Informatik, Universität Mannheim, D-68131 Mannheim

E-mail address: steidl@kiwi.math.uni-mannheim.de

Fachbereich Mathematik, Universität Rostock, D-18051 Rostock

E-mail address: manfred.tasche@mathematik.uni-rostock.de 